\title{
203.
}

\section{ON THE THEORY OF THE ANALYTICAL FORMS CALLED TREES.}

[From the Philosophical Magazine, vol. xIII. (18577), pp. 172-176.]

A symbol such as $A \partial_{x}+B \partial_{y}+\ldots$, where $A, B$, \&c. contain the variables $x, y$, \&c. in respect to which the differentiations are to be performed, partakes of the natures of an operand and operator, and may be therefore called an Operandator. Let $P, Q, R, \ldots$ be any operandators, and let $U$ be a symbol of the same kind, or to fix the ideas, a mere operand; $P U$ denotes the result of the operation $P$ performed on $U$, and $Q P U$ denotes the result of the operation $Q$ performed on $P U$; and generally in such combinations of symbols, each operation is considered as affecting the operand denoted by means of all the symbols on the right of the operation in question. Now considering the expression $Q P U$, it is easy to see that we may write

$$
Q P U=(Q \times P) U+(Q P) U
$$

where on the right-hand side $(Q \times P)$ and $(Q P)$ signify as follows: viz. $Q \times P$ denotes the mere algebraical product of $Q$ and $P$, while $Q P$ (consistently with the general notation as before explained) denotes the result of the operation $Q$ performed upon $P$ as operand; and the two parts $(Q \times P) U$ and $(Q P) U$ denote respectively the results of the operations $(Q \times P)$ and $(Q P)$ performed each of them upon $U$ as operand. It is proper to remark that $(Q \times P)$ and $(P \times Q)$ have precisely the same meaning; and the symbol may be written in either form indifferently. But without a more convenient notation, it would be difficult to find the corresponding expressions for $R Q P U$, \&c. This, however, can be at once effected by means of the analytical forms called trees (see figs. 1, 2, 3), which contain all the trees which can be formed with one branch, two branches, and three branches respectively. 
The inspection of these figures will at once show what is meant by the term in question, and by the terms root, branches (which may be either main branches, intermediate branches, or free branches), and knots (which may be either the root itself, or proper knots, or the extremities of the free branches). To apply this to the question in hand, $P U$ consists of a single term represented by fig. 1 (bis); $Q P U$ consists, as above, of two terms represented by the two parts of fig. 2 (bis), viz. the

Fig. 1. Fig. 2.

Fig. 3.
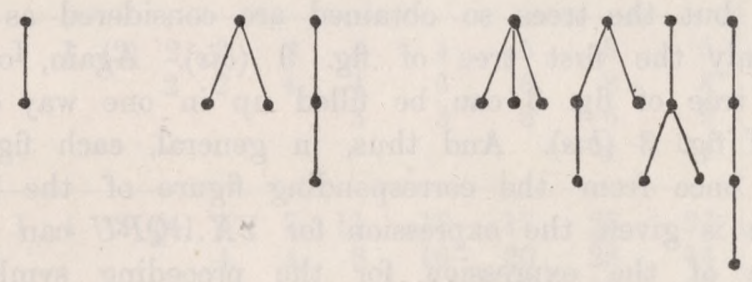

first part represents the term $(Q \times P) U$, and the second part represents the term $(Q P) U$. And it is obvious that fig. 2 (bis) is at once formed from the figure 1 (bis) by adding on a branch terminated by $Q$ at each of the knots of the single part of fig. 1 (bis). In like manner $R Q P U$ consists of six terms represented by the six parts of fig. $3($ bis $)$, and this figure is at once formed from fig. 2 (bis) by adding on a branch terminated by $R$ at each knot of each part of fig. 2 (bis). It is

Fig. 1 (bis). Fig. 2 (bis).

Fig. 3 (bis).
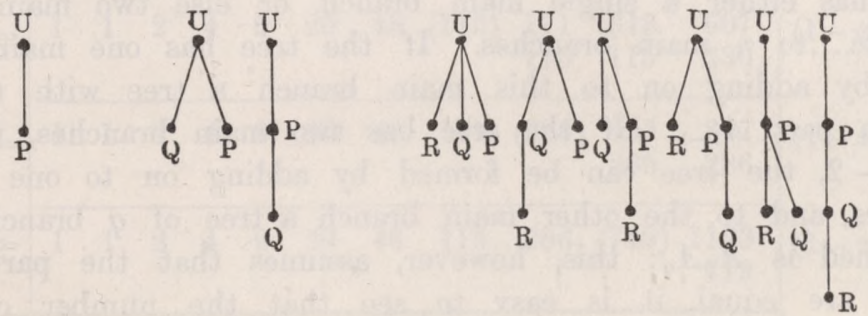

hardly necessary to remark that the first part of fig. 3 (bis) denotes what, in the notation first explained, would be denoted by $(R \times Q \times P) U$, the second term what would in like manner be denoted by $(R Q \times P) U$, and so on, the last part being the term which would be denoted by $((R Q) P) U$; viz. $R$ operates upon $Q$, giving the operandator $R Q$, which operates upon $P$, giving the operandator $(R Q) P$, which finally operates upon $U$.

The figures 1 (bis), 2 (bis), \&c. contain the same trees as are contained in the corresponding figures 1, 2, \&c.; only, on account of the different modes of filling up, trees are considered as so many distinct trees in a figure of the second set which are considered as one and the same tree in the corresponding figure of the first set. A difference in the number of trees first occurs in the figures 3 and 3 (bis), the 
first of which contains only four, while the latter contains six trees, viz. the first tree, the second, third and fourth trees, the fifth tree and the sixth tree of fig. 3 (bis) correspond respectively to the first tree, the second tree, the third tree, and the fourth tree of fig. 3. To derive fig. 3 (bis) from fig. 3, we must fill up the trees of fig. 3 with $U$ at the root and $R, Q, P$ at the other knots in every possible manner, subject only to the restriction, that, reckoning up from the extremity of a free branch to the root, there must not be any transposition in the order of the symbols $R Q P$, and taking care to admit only distinct trees. Thus the first tree of fig. 3 might be filled up in six ways; but the trees so obtained are considered as one and the same tree, and we have only the first tree of fig. 3 (bis). Again, on account of the restriction, the fourth tree of fig. 3 can be filled up in one way only, and we have thus the sixth tree of fig. 3 (bis). And thus, in general, each figure of the second set can be formed at once from the corresponding figure of the first set; or when the first set of figures is given, the expression for $Y X \ldots Q P U$ can be formed directly without the assistance of the expression for the preceding symbol $X \ldots Q P U$; the number of terms for the $n$th figure of the second set is obviously $1.2 .3 \ldots n$, and consequently it is only necessary to count the terms in order to ascertain that no admissible mode of filling up has been omitted.

The number of parts in any one of the figures of the first set is much smaller than the number of parts in the corresponding figure of the second set; and the law for the number of parts, i.e. for the number $A_{n}$ of the trees with $n$ branches, is a very singular one. To obtain this law, we must consider how the trees with $n$ branches can be formed by means of those of a smaller number of branches. A tree with $n$ branches has either a single main branch, or else two main branches, three main branches, \&c....to $n$ main branches. If the tree has one main branch, it can only be formed by adding on to this main branch a tree with $(n-1)$ branches, i.e. $A_{n}$ contains a part $A_{n-1}$. If the tree has two main branches, then $p+q$ being a partition of $n-2$, the tree can be formed by adding on to one main branch a tree of $p$ branches, and to the other main branch a tree of $q$ branches; the number of trees so obtained is $A_{p} A_{q}$ : this, however, assumes that the parts $p$ and $q$ are unequal; if they are equal, it is easy to see that the number of trees is only $\frac{1}{2} A_{p}\left(A_{p}+1\right)$. Hence $p+q$ being any partition of $n-2, A_{n}$ contains the part $A_{p} A_{q}$ if $p$ and $q$ are unequal, and the part $\frac{1}{2} A_{p}\left(A_{p}+1\right)$ if $p$ and $q$ are equal. In like manner, considering the trees with three main branches, then if $p+q+r$ is any partition of $n-3, A_{n}$ contains the part $A_{p} A_{q} A_{r}$ if $p, q, r$ are unequal; but if two of these numbers, e.g. $p$ and $q$, are equal, then the part $\frac{1}{2} A_{p}\left(A_{p}+1\right) A_{r}$; and if $p, q, r$ are all equal, then the part $\frac{1}{6} A_{p}\left(A_{p}+1\right)\left(A_{p}+2\right)$; and so on, until lastly we have a single tree with $n$ main branches, or $A_{n}$ contains the part unity. A little consideration will show that the preceding rule for the formation of the number $A_{n}$ is completely expressed by the equation

$$
(1-x)^{-1}\left(1-x^{2}\right)^{-A_{1}}\left(1-x^{3}\right)^{-A_{2}}\left(1-x^{4}\right)^{-A_{3}} \ldots=1+A_{1} x+A_{2} x^{2}+A_{3} x^{3}+A_{4} x^{4}+\& c .,
$$

and consequently that we may, by means of this equation, calculate successively for the different values of $n$ the number $A_{n}$ of the trees with $n$ branches. The calculation 
may be effected very easily as follows: [the table as originally printed contained at the end of it some errors of calculation which were corrected, B.A. Report for 1875, p. 258].

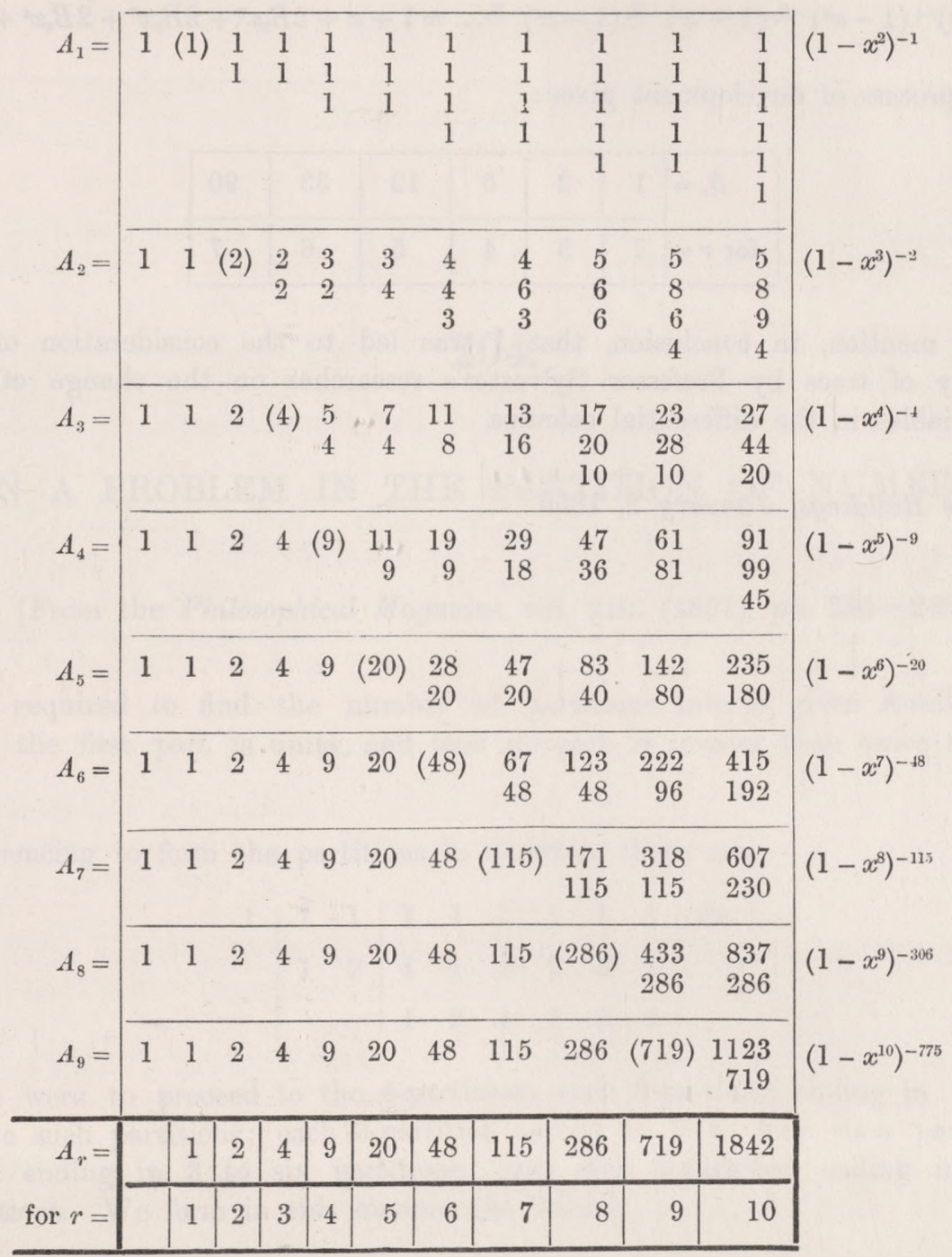

I have had occasion, for another purpose, to consider the question of finding the number of trees with a given number of free branches, bifurcations at least. Thus, when the number of free branches is three, the trees of the form in question are Fig.

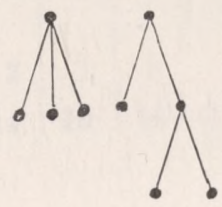


those in the annexed figure, and the number is therefore two. It is not difficult to see that we have in this case ( $B_{r}$ being the number of such trees with $r$ free branches),

$$
(1-x)^{-1}\left(1-x^{2}\right)^{-B_{2}}\left(1-x^{3}\right)^{-B_{3}}\left(1-x^{4}\right)^{-B_{4}} \ldots=1+x+2 B_{2} x^{2}+2 B_{3} x^{3}+2 B_{4} x^{4}+\& \mathrm{c} .
$$

and a like process of development gives:

\begin{tabular}{|c|c|c|c|c|c|c|}
\hline$B_{r}=$ & 1 & 2 & 5 & 12 & 33 & 90 \\
\hline for $r$ & 2 & 3 & 4 & 5 & 6 & 7 \\
\hline
\end{tabular}

I may mention, in conclusion, that I was led to the consideration of the foregoing theory of trees by Professor Sylvester's researches on the change of the independent variables in the differential calculus.

2, Stone Buildings, January 2, 1856. 